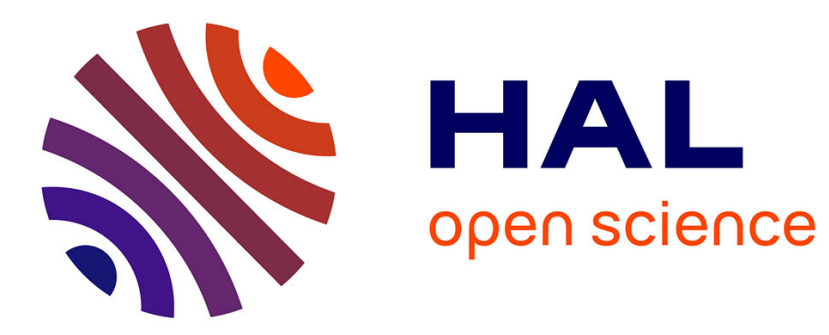

\title{
Extension of the Vook-Witt and inverse Vook-Witt elastic grain-interaction models to general loading states
}

\author{
U. Welzel, Sylvain Fréour
}

\section{To cite this version:}

U. Welzel, Sylvain Fréour. Extension of the Vook-Witt and inverse Vook-Witt elastic graininteraction models to general loading states. Philosophical Magazine, 2007, 87 (26), pp.3921-3943. 10.1080/14786430701203176 . hal-01006887

\section{HAL Id: hal-01006887 \\ https://hal.science/hal-01006887}

Submitted on 8 Dec 2017

HAL is a multi-disciplinary open access archive for the deposit and dissemination of scientific research documents, whether they are published or not. The documents may come from teaching and research institutions in France or abroad, or from public or private research centers.
L'archive ouverte pluridisciplinaire HAL, est destinée au dépôt et à la diffusion de documents scientifiques de niveau recherche, publiés ou non, émanant des établissements d'enseignement et de recherche français ou étrangers, des laboratoires publics ou privés. 


\title{
Extension of the Vook-Witt and inverse Vook-Witt elastic grain-interaction models to general loading states
}

\author{
U. WELZEL* and S. FRÉOUR $†$ \\ Max Planck Institute for Metals Research, Heisenbergstraße 3, \\ D-70569 Stuttgart, Germany
}

The recently developed Vook-Witt and inverse Vook-Witt elastic graininteraction models have been employed for the calculation of mechanical elastic constants and diffraction (X-ray) stress factors of, in particular, thin films. However, their applicability is limited to a planar, rotationally symmetric state of macroscopic, mechanical stress. For such a loading state (and an, at least, transversely, elastically isotropic specimen), only two mechanical elastic constants are necessary to describe mechanical elastic behaviour and only the sum of two diffraction (X-ray) stress factors is needed to relate lattice strains to the one independent component of the mechanical stress tensor. The restriction to a planar, rotationally symmetric state of mechanical stress will be removed in this work. Calculation of the full stiffness tensor and all six diffraction (X-ray) stress factors then becomes possible. It was found previously that the Vook-Witt and inverse Vook-Witt models become (but only approximately) equivalent to the Eshelby-Kröner model for certain ideal grain-shape textures. For this reason, results of numerical calculations of mechanical elastic constants and diffraction (X-ray) stress factors, based on the Vook-Witt and inverse Vook-Witt models, will be presented and compared to corresponding results obtained from the Eshelby-Kröner grain-interaction model considering ideal grain-shape ('morphological') textures.

\section{Introduction}

For polycrystals, the macroscopic, mechanical elastic constants (relating mechanical strains to mechanical stresses) and the so-called diffraction (X-ray) elastic constants (diffraction stress factors for elastically anisotropic samples, relating lattice strains to mechanical stresses) can be calculated from single-crystal elastic data of individual grains composing the polycrystals [1-11]. To this end so-called elastic graininteraction models, describing the distribution of stresses and strains over the differently oriented grains, can be employed [3-6].

*Corresponding author. Email: u.welzel@mf.mpg.de.

$\dagger$ Present address: Institut de Recherche en Génie-Civil et Mécanique, GeM-CRTT, 37 Bd. de l'Université, 44602 Saint-Nazaire, France 
Traditionally, isotropic grain-interaction models are considered, where the same grain-interaction assumptions are adopted for all directions in the specimen [4]. A polycrystal with isotropic grain interaction is macroscopically, elastically isotropic (sometimes termed quasi-isotropic) in the absence of crystallographic texture, whereas macroscopic elastic anisotropy occurs in the presence of crystallographic texture. The Voigt [7], Reuss [8], Neerfeld-Hill [9, 10] models and the Eshelby-Kröner model (for polycrystals consisting of, on the average, spherical grains) [11] fall in this category.

However, even in the absence of crystallographic texture, polycrystals cannot generally be considered as being mechanically, elastically isotropic. Thin films provide a possible example because, due to their reduced dimensionality, thin films can exhibit transverse elastic isotropy (only) even in the absence of crystallographic texture. Recently, it has been demonstrated theoretically and experimentally by van Leeuwen et al. [12] that the application of a new type of grain-interaction model, a direction-dependent grain-interaction model, is compatible with macroscopic, anisotropic elastic behaviour, even in the absence of crystallographic texture [13-15]. The notion "direction-dependent grain interaction" signifies that different graininteraction assumptions prevail along different directions in the specimen. In the Vook-Witt model, explored by van Leeuwen et al. [12] (cf. also [16]), for example, a Reuss-type grain interaction (i.e. certain stress tensor components are identical for all crystallites) is adopted for the out-of-plane components of the stress tensor, whereas a Voigt-type grain interaction (i.e. certain strain tensor components are identical for all crystallites) is adopted for all in-plane components of the strain tensor. As extreme grain-interaction assumptions are adopted in the Vook-Witt model, it cannot be expected to be fully compatible with physical reality. To overcome this problem, the inverse Vook-Witt model, required for the construction of an effective grain-interaction model, has been recently proposed [14, 15].

In thin films, planar, rotationally symmetric states of residual stress are frequently met. However, other stress states may occur, in particular, in cases of applied stress. So far, the Vook-Witt and inverse Vook-Witt models have only been applied to a planar rotationally symmetric state of stress. This restriction will be removed in this work by a generalization of the grain-interaction assumptions. This generalization also enables the calculation of the full mechanical stiffness tensor (i.e. of five independent tensor components), whereas previously, only two mechanical elastic constants could be calculated. Moreover, all six diffraction (X-ray) stress factors can now be calculated, whereas previously, only the sum of two diffraction stress factors was accessible.

The results for mechanical elastic constants and diffraction stress factors will be compared to results from the Eshelby-Kröner model for specimens with an ideal grain-shape texture (see [17] for a detailed discussion of the calculation scheme). In earlier investigations, similarities between the mechanical and diffraction elastic constants, calculated using the Vook-Witt and Eshelby-Kröner models, have been shown in the case of a polycrystal consisting of flat-disc shaped grains. Analogous similarities occur (but much less outspokenly) with the inverse Vook-Witt model and Eshelby-Kröner model in the case of a polycrystal consisting of needle-shaped grains [5]. Analogous findings are obtained in this work. 


\section{Theoretical background}

\subsection{Conventions}

2.1.1. Frames of reference and transformation of tensors from one frame of reference to another. Stresses, strains and elastic constants are tensorial quantities and it is, thus, necessary to define frames of reference. It is convenient to employ the following three Cartesian frames of reference: the crystal frame of reference $(C)$, the specimen frame of reference $(S)$ and the laboratory frame of reference $(L)$. For details on the definition of these frames of reference, see, for example, [13] (for a definition of the crystal frame of reference in the case of non-cubic crystallites, see also [18]). A superscript $(C, S$ or $L)$ will be attached to a tensor to indicate the reference frame adopted for the representation of the tensor. The absence of any superscript implies the validity of an equation independent of the reference frame used for tensor representation, but the same reference frame has to be adopted for all tensors in the equation. Figure 1 shows the relative orientation of the specimen and laboratory frame of references, and a definition of angles $\psi$ and $\varphi$, where $\psi$ is the inclination angle of the diffraction vector with respect to the sample surface normal and $\varphi$ denotes the rotation of the sample around the sample surface normal.

Transformations of tensors from one frame of reference to another can be performed employing rotation matrices. For a detailed description of the rotation matrices required for performing calculations described in this paper, see [13] for the case of cubic crystal symmetry and consider the appendix of [17] for cases of lower

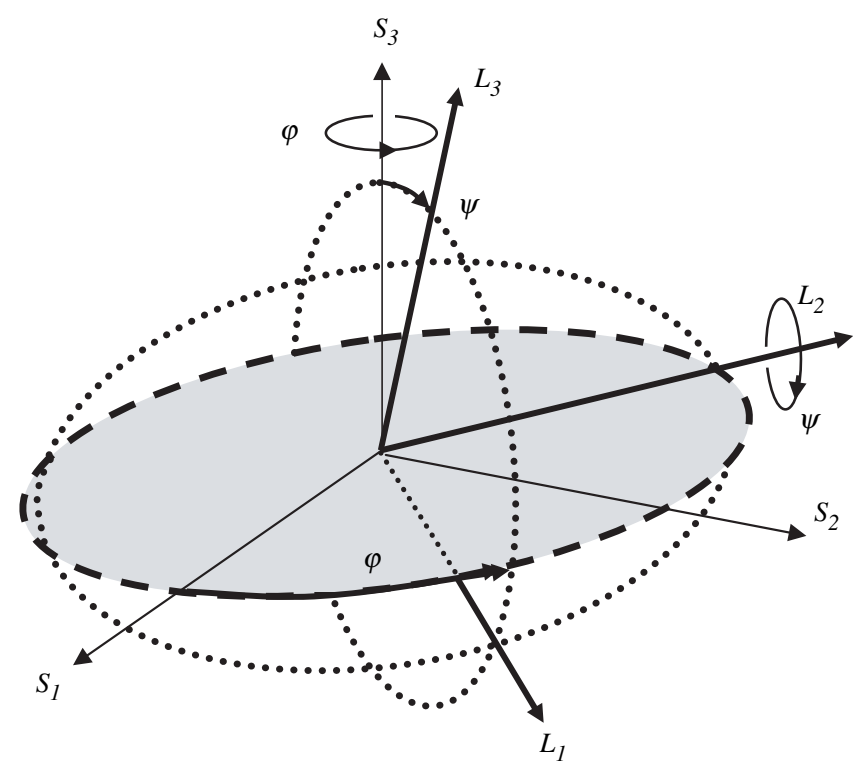

Figure 1. Definition of and relationship between the sample $(S)$ and laboratory $(L)$ reference frames. 
crystal symmetry. For a general introduction to the use of transformation matrices, see [3] and [18].

2.1.2. Calculation of averages. In the following, a single-phase polycrystal is considered, which may be crystallographically textured. It is useful to distinguish three types of averages of a tensor $\boldsymbol{\Omega}$ (as, for example, the strain tensor $\boldsymbol{\varepsilon}$ ):

- Averages of a tensor $\boldsymbol{\Omega}$ for all grains having the same crystallographic orientation in the volume considered (the notion "mesoscopic scale" is also used to denote such averages [19]). This average will be denoted by the symbol $\mathbf{\Omega}(\mathbf{g})$, where $\mathbf{g}$ represents a vector in the three-dimensional crystallographic orientation (Euler) space $G$ and defines the crystallographic orientation (for details, see [13]). $\mathbf{g}=(\alpha, \beta, \gamma)$, where $\alpha, \beta$ and $\gamma$ are three Euler angles. The convention of Roe and Krigbaum [20] for the definition of the Euler angles will be adopted.

- Averages of a tensor $\boldsymbol{\Omega}$ for all crystallographically, differently oriented grains in the volume considered (so-called macroscopic, mechanical averages). This average will be denoted by angular brackets $\langle\Omega\rangle$ and can be calculated as follows:

$$
\langle\mathbf{\Omega}\rangle=\frac{1}{8 \pi^{2}} \int_{G} f(\mathbf{g}) \mathbf{\Omega}(\mathbf{g}) \mathrm{d}^{3} \mathbf{g}
$$

where $f(\mathbf{g})$, the crystallographic orientation distribution function (CODF), has to be employed as a weighting function where crystallographic texture occurs (for details, see [13]). The factor $1 /\left(8 \pi^{2}\right)$ serves as a normalization factor and is the volume of the Euler space $G$.

- Averages of a tensor $\boldsymbol{\Omega}$ for the diffracting crystallites in a diffraction measurement. As the orientation of the diffracting crystallites differs only by a rotation around the diffraction vector, the averaging has to be performed with respect to this degree of freedom, in the following called $\lambda$ (for details, see [13]):

$$
\{\boldsymbol{\Omega}\}_{\varphi, \psi}^{h k l}=\frac{\int_{0}^{2 \pi} \boldsymbol{\Omega}(h k l, \lambda, \varphi, \psi) f^{*}(h k l, \lambda, \varphi, \psi) \mathrm{d} \lambda}{\int_{0}^{2 \pi} f^{*}(h k l, \lambda, \varphi, \psi) \mathrm{d} \lambda} .
$$

$f^{*}(h k l, \lambda, \varphi, \psi)$ denotes the representation of the CODF in terms of the measurement parameters and the rotation angle with respect to the diffraction vector. The CODF, as used in equation (1), cannot be directly used in equation (2) since the angles $\lambda, \varphi, \psi$ are not Euler angles representing a rotation of the $C$ system with respect to the $S$ system (they provide the rotation of the system $L$ with respect to the system $S$ ). However, the values of $\alpha, \beta, \gamma$ and, thus, $f(\alpha, \beta, \gamma)$ at every $\lambda$ can be calculated from $h k l, \lambda, \varphi$ and $\psi$, to be finally substituted for $f^{*}(h k l, \lambda, \varphi, \psi)$ in equation (2) (for a more detailed treatment of the necessary calculations, see [13]). 
2.1.3. Lattice strains and diffraction stress factors. The lattice strain for a particular reflection $h k l$ at the orientation of the diffraction vector $(\varphi, \psi)$ can be calculated according to (cf. equation (2)):

$$
\varepsilon(h k l, \varphi, \psi)=\left\{\varepsilon_{33}^{L}\right\}_{\varphi, \psi}^{h k l}=\frac{\int_{0}^{2 \pi} \varepsilon_{33}^{L}(h k l, \lambda, \varphi, \psi) f^{*}(h k l, \lambda, \varphi, \psi) \mathrm{d} \lambda}{\int_{0}^{2 \pi} f^{*}(h k l, \lambda, \varphi, \psi) \mathrm{d} \lambda} .
$$

The $\varphi, \psi$ and $h k l$-dependent diffraction (X-ray) stress factors, $F_{i j}(\psi, \varphi, h k l)$, relate the lattice strain to mechanical stress expressed in the specimen frame of reference [4]:

$$
\varepsilon(h k l, \varphi, \psi)=F_{i j}(\psi, \varphi, h k l)\left\langle\sigma_{i j}^{S}\right\rangle .
$$

2.1.4. Grain-shape/morphological texture. For the calculation of mechanical and diffraction elastic constants of polycrystals with a grain-shape texture employing the Eshelby-Kröner model, the treatment will be restricted to polycrystals consisting of ellipsoidal grains. $\uparrow$ It will be assumed that the ellipsoidal grains exhibit identical orientations of their principal shape axes in the specimen frame of reference, i.e. an ideal grain-shape texture occurs. An ideal grain-shape texture is considered in the following as, only in this case, unique mechanical elastic constants and X-ray stress factors can be calculated employing the Eshelby-Kröner model (for a more detailed discussion of the effect of a non-ideal grain-shape texture, see [17]).

Note that the principal axes of a grain are only related to the external (geometrical) shape of the grain. Thereby, nothing is prescribed regarding the crystallographic orientation of the grain (crystallite). In general, the (ellipsoidal) grains constituting the specimen will have different crystallographic orientations.

The shape of the grains will be described by a shape parameter $\eta$, which is defined as the ratio of the principal axis of the ellipsoid in the $z$-direction $\left(a_{3}\right)$ of the specimen frame of reference to the principal axes of the ellipsoid in the $x$-direction $\left(a_{1}\right)$ and the $y$-direction $\left(a_{2}\right)$ in the specimen frame of reference, respectively:

$$
\eta=\frac{a_{3}}{a_{1}}=\frac{a_{3}}{a_{2}} .
$$

Thus, the considered ellipsoidal grains present rotational symmetry with respect to the surface normal of the specimen.

$\dagger$ Of course, a real polycrystal cannot consist of ellipsoidal grains (only). The ellipsoidal shape is an idealized shape, which is adopted to represent grains with (average) aspect ratios different from 1, whereas a spherical shape is adopted for grains with an (average) aspect ratio of 1 . 


\section{Generalization of the Vook-Witt and inverse Vook-Witt models to any elastic loading state}

\subsection{Vook-Witt model: generalized grain-interaction assumptions}

Traditionally, an, at least, transversely, elastically isotropic specimen and a planar, rotationally symmetric mechanical state of stress are considered for the Vook-Witt (VW) model:

$$
\begin{gathered}
\left\langle\boldsymbol{\varepsilon}^{S}\right\rangle=\left(\begin{array}{ccc}
\varepsilon_{\|}^{S} & 0 & 0 \\
0 & \varepsilon_{\|}^{S} & 0 \\
0 & 0 & \varepsilon_{\perp}^{S}
\end{array}\right), \\
\left\langle\boldsymbol{\sigma}^{S}\right\rangle=\left(\begin{array}{ccc}
\sigma_{\|}^{S} & 0 & 0 \\
0 & \sigma_{\|}^{S} & 0 \\
0 & 0 & 0
\end{array}\right) .
\end{gathered}
$$

The following grain-interaction assumptions are adopted: (i) the strain is rotationally symmetric in the plane of the film and (ii) equal for all crystallites, and (iii) the stresses perpendicular to the layer are zero for all crystallites, i.e. the crystallites can deform freely in this direction. Recognizing the symmetry of the stress and strain tensors, these assumptions fix parts of the stress and strain tensor for all crystallites (on the mesoscopic scale; cf. section 2.1) [12, 13]:

$$
\begin{gathered}
\boldsymbol{\varepsilon}^{s}=\left(\begin{array}{ccc}
\varepsilon_{\|}^{S} & 0 & \diamond \\
0 & \varepsilon_{\|}^{S} & \diamond \\
\diamond & \diamond & \diamond
\end{array}\right), \\
\boldsymbol{\sigma}^{s}=\left(\begin{array}{lll}
\diamond & \diamond & 0 \\
\diamond & \diamond & 0 \\
0 & 0 & 0
\end{array}\right) .
\end{gathered}
$$

The tensor components marked by $\diamond$ are not explicitly specified for every crystallite, but these components can be calculated from Hooke's law for every crystallite:

$$
\varepsilon_{i j}^{S}=s_{i j k l}^{S} \sigma_{k l}^{S} .
$$

The $s_{i j k l}^{S}$ are the single crystal elastic compliances expressed in the specimen frame of reference. Equation (10) represents a system of nine equations for 18 unknowns, but, as the strain and stress tensors are symmetric (i.e. $\varepsilon_{i j}=\varepsilon_{j i}$ and $\sigma_{i j}=\sigma_{j i}$ ), equation (10) is a short notation for six independent equations for 12 independent unknowns. If six components of the 12 unknowns are known, as a consequence, the other components can be calculated by solving the system of equation (10).

A generalization of the Vook-Witt model to arbitrary loading states can be achieved by adopting the following grain-interaction assumptions:

$$
\boldsymbol{\varepsilon}^{S}=\left(\begin{array}{ccc}
\left\langle\varepsilon_{11}^{S}\right\rangle & \left\langle\varepsilon_{12}^{S}\right\rangle & \diamond \\
\left\langle\varepsilon_{12}^{S}\right\rangle & \left\langle\varepsilon_{22}^{S}\right\rangle & \diamond \\
\diamond & \diamond & \diamond
\end{array}\right),
$$




$$
\boldsymbol{\sigma}^{S}=\left(\begin{array}{ccc}
\diamond & \diamond & \left\langle\sigma_{13}^{S}\right\rangle \\
\diamond & \diamond & \left\langle\sigma_{23}^{S}\right\rangle \\
\left\langle\sigma_{13}^{S}\right\rangle & \left\langle\sigma_{23}^{S}\right\rangle & \left\langle\sigma_{33}^{S}\right\rangle
\end{array}\right) .
$$

Note that the \langle\rangle brackets indicate that a tensor component is taken equal to the tensor component of the corresponding mechanical stress or strain tensor. The tensor components marked by $\diamond$ are not explicitly specified for every crystallite, but these components can be calculated from Hooke's law for every crystallite (equation (10); for details, see Appendix A).

The above formulated grain-interaction assumptions (equations (11) and (12)) imply that the polycrystal is (at least) transversely, elastically isotropic in the absence of crystallographic texture, as the same grain interaction assumptions (equality of strains) are adopted for all directions parallel to the surface.

\subsection{Inverse Vook-Witt model: generalized grain-interaction assumptions}

In the inverse Vook-Witt (iVW) model, the traditionally adopted grain-interaction assumptions for a transversely isotropic specimen subjected to a plane, rotationally symmetric state of stress (cf. equation (7)) are as follows: (i) the in-plane stress is rotationally symmetric and (ii) equal for all crystallites, and (iii) the strain perpendicular to the film surface is equal for all crystallites [14, 15]. As in the (regular) VW model, recognizing the symmetry of the stress and strain tensors, these assumptions fix certain strain and stress tensor components for all crystallites (on the mesoscopic scale; cf. section 2.1) [14, 15]:

$$
\begin{aligned}
\boldsymbol{\varepsilon}^{S} & =\left(\begin{array}{ccc}
\diamond & \diamond & 0 \\
\diamond & \diamond & 0 \\
0 & 0 & \varepsilon_{\perp}^{S}
\end{array}\right), \\
\boldsymbol{\sigma}^{S} & =\left(\begin{array}{ccc}
\sigma_{\|}^{S} & 0 & \diamond \\
0 & \sigma_{\|}^{S} & \diamond \\
\diamond & \diamond & \diamond
\end{array}\right) .
\end{aligned}
$$

The missing strain and stress tensor components for each crystallite, marked by $\diamond$, can, analogous to the VW model, be calculated from Hooke's law (cf. discussion in section 3.1).

A generalization of the iVW model to arbitrary loading states can be achieved by adopting the following grain-interaction assumptions:

$$
\begin{aligned}
\boldsymbol{\varepsilon}^{S}= & \left(\begin{array}{ccc}
\diamond & \diamond & \left\langle\varepsilon_{13}^{S}\right\rangle \\
\diamond & \diamond & \left\langle\varepsilon_{23}^{S}\right\rangle \\
\left\langle\varepsilon_{13}^{S}\right\rangle & \left\langle\varepsilon_{23}^{S}\right\rangle & \left\langle\varepsilon_{33}^{S}\right\rangle
\end{array}\right), \\
\boldsymbol{\sigma}^{S} & =\left(\begin{array}{ccc}
\left\langle\sigma_{11}^{S}\right\rangle & \left\langle\sigma_{12}^{S}\right\rangle & \diamond \\
\left\langle\sigma_{12}^{S}\right\rangle & \left\langle\sigma_{22}^{S}\right\rangle & \diamond \\
\diamond & \diamond & \diamond
\end{array}\right) .
\end{aligned}
$$


Note that the \langle\rangle brackets indicate that a tensor component is taken equal to the tensor component of the corresponding mechanical stress or strain tensor, respectively. The tensor components marked by $\diamond$ are not explicitly specified for every crystallite, but these components can be calculated from Hooke's law for every crystallite (equation (10); for details, see Appendix A).

Note that the above formulated grain-interaction assumptions (equations (15) and (16)) imply that the polycrystal is (at least) transversely, elastically isotropic in the absence of crystallographic texture as the same grain interaction assumptions (equality of stresses) are adopted for all directions parallel to the surface.

\subsection{Calculation of the mechanical elastic constants}

Under the condition of a plane, rotationally symmetric state of mechanical stress, frequently met in thin films, (cf. equations (6) and (7)), two mechanical elastic constants $A$ and $B$ suffice, as only three independent non-zero stress and strain tensor components occur:

$$
\begin{gathered}
\varepsilon_{\|}^{S}=A \sigma_{\|}^{S}, \\
\varepsilon_{\perp}^{S}=B \sigma_{\|}^{S} .
\end{gathered}
$$

Only these two mechanical elastic constants $A$ and $B$ were accessible, so far, employing the VW and iVW models. Using the generalization of the graininteraction assumptions adopted in the present work (cf. equations (11) and (12) for case of the VW model and equations (15) and (16) for the case of the iVW model), calculation of the full mechanical stiffness tensor is now possible by considering a set of hypothetical mechanical loading states. The procedure outlined below presupposes that the polycrystal is (at least) mechanically, transversely elastically isotropic. This is the case if the polycrystal is untextured or fibre-textured, with the fibre axis coinciding with the $z$-axis of the specimen frame of reference (cf. sections 3.1 and 3.2). For the case of crystallographic texture with a lower symmetry, more than five independent components of the mechanical stiffness (and compliance) tensor can occur. Then, more than three loading states have to be considered for the calculation of all independent stiffness tensor components (see Appendix B, for an example).

The following mechanical loading states will be considered: (i) uniaxial strain along the $S_{1}$-axis, (ii) uniaxial strain along the $S_{3}$-axis and (iii) pure shear-strain loading:

(i) Uniaxial strain along the $S_{1}$-axis:

$$
\left\langle\boldsymbol{\varepsilon}^{S}\right\rangle=\left(\begin{array}{ccc}
\left\langle\varepsilon_{11}^{S}\right\rangle & 0 & 0 \\
0 & 0 & 0 \\
0 & 0 & 0
\end{array}\right),
$$




$$
\left\langle\boldsymbol{\sigma}^{S}\right\rangle=\left(\begin{array}{ccc}
\left\langle\sigma_{11}^{S}\right\rangle & 0 & 0 \\
0 & \left\langle\sigma_{22}^{S}\right\rangle & 0 \\
0 & 0 & \left\langle\sigma_{33}^{S}\right\rangle
\end{array}\right) .
$$

For the VW model, the grain-interaction assumptions read (cf. equations (11) and (12)):

$$
\begin{aligned}
\boldsymbol{\varepsilon}^{S} & =\left(\begin{array}{ccc}
\left\langle\varepsilon_{11}^{S}\right\rangle & 0 & \diamond \\
0 & 0 & \diamond \\
\diamond & \diamond & \diamond
\end{array}\right), \\
\boldsymbol{\sigma}^{s} & =\left(\begin{array}{ccc}
\diamond & \diamond & 0 \\
\diamond & \diamond & 0 \\
0 & 0 & \left\langle\sigma_{33}^{S}\right\rangle
\end{array}\right) .
\end{aligned}
$$

For a given strain $\left\langle\varepsilon_{11}^{S}\right\rangle$, a certain value has to be assigned to $\left\langle\sigma_{33}^{S}\right\rangle$ to realize a uniaxial strain state, i.e. to ensure that $\left\langle\varepsilon_{33}^{S}\right\rangle=0$. Thus, the following procedure can be followed: starting from arbitrary values for the strain $\left\langle\varepsilon_{11}^{S}\right\rangle$ and $\left\langle\sigma_{33}^{S}\right\rangle$, the mechanical averages of the strain and stress tensors can be calculated. In general, the strain perpendicular to the surface, $\left\langle\varepsilon_{33}^{S}\right\rangle$, will not be zero. However, by changing the mechanical stress perpendicular to the surface $\left\langle\sigma_{33}^{S}\right\rangle$ (keeping $\left\langle\varepsilon_{11}^{S}\right\rangle$ fixed), $\left\langle\varepsilon_{33}^{S}\right\rangle$ can be made zero, so that a uniaxial strain state is achieved. Thus, the above described calculation has to be repeated for another value of $\left\langle\sigma_{33}^{S}\right\rangle$. As $\left\langle\varepsilon_{33}^{S}\right\rangle$ is linearly related to $\left\langle\sigma_{33}^{S}\right\rangle$,

$$
\left\langle\varepsilon_{33}^{S}\right\rangle=m \cdot\left\langle\sigma_{33}^{S}\right\rangle+c .
$$

The stress making $\left\langle\varepsilon_{33}^{S}\right\rangle$ equal to zero can be determined from equation (23) once the constants $m$ and $c$ have been calculated from the two (arbitrary) values of the stress $\left\langle\sigma_{33}^{S}\right\rangle_{1},\left\langle\sigma_{33}^{S}\right\rangle_{2}$ and the corresponding stresses perpendicular to the film surface $\left\langle\varepsilon_{33}^{S}\right\rangle_{1}$ and $\left\langle\varepsilon_{33}^{S}\right\rangle_{2}$. Alternatively, $\left\langle\sigma_{33}^{S}\right\rangle$ could be kept fixed and an appropriate value for $\left\langle\varepsilon_{11}^{S}\right\rangle$, for which $\left\langle\varepsilon_{33}^{S}\right\rangle=0$, could be determined following a procedure analogous to the above scheme.

For the iVW model, the grain-interaction assumptions read (cf. equations (15) and (16)):

$$
\begin{gathered}
\boldsymbol{\varepsilon}^{s}=\left(\begin{array}{ccc}
\diamond & \diamond & 0 \\
\diamond & \diamond & 0 \\
0 & 0 & 0
\end{array}\right), \\
\boldsymbol{\sigma}^{s}=\left(\begin{array}{ccc}
\left\langle\sigma_{11}^{S}\right\rangle & 0 & \diamond \\
0 & \left\langle\sigma_{22}^{S}\right\rangle & \diamond \\
\diamond & \diamond & \diamond
\end{array}\right) .
\end{gathered}
$$

For a given stress $\left\langle\sigma_{11}^{S}\right\rangle$, a certain value has to be assigned to $\left\langle\sigma_{22}^{S}\right\rangle$ (or vice versa) to realize a uniaxial strain state. To ensure that $\left\langle\varepsilon_{22}^{S}\right\rangle=0$, a procedure analogous to the procedure described below equations (21) and (22) can be followed to find an appropriate value for $\left\langle\sigma_{22}^{S}\right\rangle$. 
Once a mechanical loading state, according to equations (19) and (20), has been achieved (recognizing the mechanical transverse isotropy), three components of the mechanical stiffness tensor $\mathbf{C}$ (in Voigt two-index notation) can be calculated from:

$$
\begin{aligned}
& C_{11}^{S}=\frac{\left\langle\sigma_{11}^{s}\right\rangle}{\left\langle\varepsilon_{11}^{s}\right\rangle}, \\
& C_{12}^{S}=\frac{\left\langle\sigma_{22}^{s}\right\rangle}{\left\langle\varepsilon_{11}^{s}\right\rangle}, \\
& C_{13}^{S}=\frac{\left\langle\sigma_{33}^{s}\right\rangle}{\left\langle\varepsilon_{11}^{s}\right\rangle} .
\end{aligned}
$$

(ii) Uniaxial strain along the $S_{3}$-axis:

$$
\begin{gathered}
\left\langle\boldsymbol{\varepsilon}^{S}\right\rangle=\left(\begin{array}{ccc}
0 & 0 & 0 \\
0 & 0 & 0 \\
0 & 0 & \left\langle\varepsilon_{33}^{S}\right\rangle
\end{array}\right), \\
\left\langle\boldsymbol{\sigma}^{S}\right\rangle=\left(\begin{array}{ccc}
\left\langle\sigma_{11}^{S}\right\rangle & 0 & 0 \\
0 & \left\langle\sigma_{11}^{S}\right\rangle & 0 \\
0 & 0 & \left\langle\sigma_{33}^{S}\right\rangle
\end{array}\right) .
\end{gathered}
$$

Note that the equality of the components $\left\langle\sigma_{11}^{S}\right\rangle$ and $\left\langle\sigma_{22}^{S}\right\rangle$ is a direct consequence of the macroscopic elastic transverse isotropy.

For the VW model, the grain-interaction assumptions read (cf. equations (11) and (12)),

$$
\begin{gathered}
\boldsymbol{\varepsilon}^{S}=\left(\begin{array}{ccc}
0 & 0 & \diamond \\
0 & 0 & \diamond \\
\diamond & \diamond & \diamond
\end{array}\right), \\
\boldsymbol{\sigma}^{S}=\left(\begin{array}{ccc}
\diamond & \diamond & 0 \\
\diamond & \diamond & 0 \\
0 & 0 & \left\langle\sigma_{33}^{S}\right\rangle
\end{array}\right) .
\end{gathered}
$$

where as for the iVW model, the grain-interaction assumptions read (cf. equations (15) and (16)):

$$
\begin{gathered}
\boldsymbol{\varepsilon}^{S}=\left(\begin{array}{ccc}
\diamond & \diamond & 0 \\
\diamond & \diamond & 0 \\
0 & 0 & \left\langle\varepsilon_{33}^{S}\right\rangle
\end{array}\right), \\
\boldsymbol{\sigma}^{S}=\left(\begin{array}{ccc}
\left\langle\sigma_{11}^{S}\right\rangle & 0 & \diamond \\
0 & \left\langle\sigma_{11}^{S}\right\rangle & \diamond \\
\diamond & \diamond & \diamond
\end{array}\right) .
\end{gathered}
$$

For the iVW model, to ensure that $\left\langle\varepsilon_{11}^{S}\right\rangle=\left\langle\varepsilon_{22}^{S}\right\rangle=0$, a procedure analogous to the procedure described below equations (21) and (22) can be followed to find an appropriate value for $\left\langle\sigma_{11}^{S}\right\rangle$ (note that no such procedure is required 
for the VW model for the loading state considered). Once a mechanical loading state specified in equations (29) and (30) has been achieved, two components of the mechanical stiffness tensor $\mathbf{C}$ can be calculated from:

$$
\begin{aligned}
& C_{13}^{S}=\frac{\left\langle\sigma_{11}^{S}\right\rangle}{\left\langle\varepsilon_{33}^{S}\right\rangle}, \\
& C_{33}^{S}=\frac{\left\langle\sigma_{33}^{S}\right\rangle}{\left\langle\varepsilon_{33}^{S}\right\rangle} .
\end{aligned}
$$

(iii) Pure shear-strain loading:

$$
\begin{aligned}
& \left\langle\boldsymbol{\varepsilon}^{S}\right\rangle=\left(\begin{array}{ccc}
0 & \left\langle\varepsilon_{12}^{S}\right\rangle & 0 \\
\left\langle\varepsilon_{12}^{S}\right\rangle & 0 & \left\langle\varepsilon_{23}^{S}\right\rangle \\
0 & \left\langle\varepsilon_{23}^{S}\right\rangle & 0
\end{array}\right), \\
& \left\langle\boldsymbol{\sigma}^{S}\right\rangle=\left(\begin{array}{ccc}
0 & \left\langle\sigma_{12}^{S}\right\rangle & 0 \\
\left\langle\sigma_{12}^{S}\right\rangle & 0 & \left\langle\sigma_{23}^{S}\right\rangle \\
0 & \left\langle\sigma_{23}^{S}\right\rangle & 0
\end{array}\right) .
\end{aligned}
$$

For the VW model, the grain-interaction assumptions read (cf. equations (11) and (12)),

$$
\begin{aligned}
\boldsymbol{\varepsilon}^{S} & =\left(\begin{array}{ccc}
0 & \left\langle\varepsilon_{12}^{S}\right\rangle & \diamond \\
\left\langle\varepsilon_{12}^{S}\right\rangle & 0 & \diamond \\
\diamond & \diamond & \diamond
\end{array}\right), \\
\boldsymbol{\sigma}^{S} & =\left(\begin{array}{ccc}
\diamond & \diamond & 0 \\
\diamond & \diamond & \left\langle\sigma_{23}^{S}\right\rangle \\
0 & \left\langle\sigma_{23}^{S}\right\rangle & 0
\end{array}\right) .
\end{aligned}
$$

For the iVW model, the grain-interaction assumptions read (cf. equations (15) and (16)):

$$
\begin{gathered}
\boldsymbol{\varepsilon}^{S}=\left(\begin{array}{ccc}
\diamond & \diamond & 0 \\
\diamond & \diamond & \left\langle\varepsilon_{23}^{S}\right\rangle \\
0 & \left\langle\varepsilon_{23}^{S}\right\rangle & 0
\end{array}\right), \\
\boldsymbol{\sigma}^{S}=\left(\begin{array}{ccc}
0 & \left\langle\sigma_{12}^{S}\right\rangle & \diamond \\
\left\langle\sigma_{12}^{S}\right\rangle & 0 & \diamond \\
\diamond & \diamond & \diamond
\end{array}\right) .
\end{gathered}
$$


Recognizing the mechanical transverse isotropy, two independent components of the mechanical stiffness tensor $\mathbf{C}$ can be calculated from:

$$
\begin{gathered}
C_{44}^{S}=C_{55}^{S}=\frac{\left\langle\sigma_{23}^{S}\right\rangle}{\left(2\left\langle\varepsilon_{23}^{S}\right\rangle\right)}, \\
C_{66}^{S}=\frac{\left\langle\sigma_{12}^{S}\right\rangle}{\left(2\left\langle\varepsilon_{12}^{S}\right\rangle\right)} .
\end{gathered}
$$

Thus, from equations (26)-(28) (or, alternatively (35)), (36), (43) and (44), the five independent components of the mechanical stiffness tensor can be calculated. Note that other sets of hypothetical loading states can be conceived, which also allow the evaluation of the full stiffness tensor.

\subsection{Calculation of lattice strains and diffraction stress factors}

In the following, a procedure will be outlined for calculating lattice strains for a given mechanical state of stress $\left\langle\boldsymbol{\sigma}^{S}\right\rangle$. The procedure runs as follows:

(i) The mechanical elastic constants, i.e. the full stiffness tensor $\mathbf{C}$ and its inverse, the mechanical compliance tensor $\mathbf{S}$, have to be calculated first, following the procedure outlined in section 3.3.

(ii) From the mechanical stress tensor $\left\langle\boldsymbol{\sigma}^{S}\right\rangle$, the mechanical strain tensor $\left\langle\boldsymbol{\varepsilon}^{S}\right\rangle$ can be calculated from Hooke's law:

$$
\left\langle\varepsilon^{S}\right\rangle=\mathbf{S}^{S}\left\langle\boldsymbol{\sigma}^{S}\right\rangle
$$

with

$$
\mathbf{S}^{S}=\left(\mathbf{C}^{S}\right)^{-1}
$$

(iii) Employing the grain-interaction assumptions (cf. equations (11) and (12) for the VW model and equations (15) and (16) for the iVW model), the strain tensor $\boldsymbol{\varepsilon}^{S}(\mathbf{g})$ and, thus, the lattice strain $\varepsilon(h k l, \varphi, \psi)$ can be calculated (cf. equation (3)).

(iv) The diffraction stress factors can be obtained by imposing mechanical stress states with only one non-zero stress tensor component:

$$
F_{i j}(\psi, \varphi, h k l)=\frac{\varepsilon(h k l, \varphi, \psi)}{\left\langle\sigma_{i j}^{S}\right\rangle} .
$$

\section{Results and discussion}

Mechanical and diffraction elastic constants of polycrystals consisting of tungsten, copper and iron are presented in the following. Single-crystal elastic data have been listed in table 1 . For cubic crystallites, an anisotropy factor $A_{i}$ can be defined as:

$$
A_{i}=2 \frac{\left(s_{11}^{C}-s_{12}^{C}\right)}{s_{44}^{C}} .
$$


Table 1. Single crystal elastic stiffnesses of cubic materials used in this work [21].

\begin{tabular}{lcccc}
\hline & $s_{11}\left(\mathrm{TPa}^{-1}\right)$ & $s_{12}\left(\mathrm{TPa}^{-1}\right)$ & $s_{44}\left(\mathrm{TPa}^{-1}\right)$ & $A_{i}$ \\
\hline $\mathrm{W}(\mathrm{bcc})$ & 2.57 & -0.73 & 6.60 & 1.00 \\
$\mathrm{Cu}(\mathrm{fcc})^{*}$ & 14.995 & -6.2822 & 13.263 & 3.21 \\
$\mathrm{Fe}(\mathrm{bcc})$ & 7.62 & -2.79 & 8.58 & 2.43 \\
\hline
\end{tabular}

${ }^{*}$ Calculated from stiffness tensor components.

Table 2. Stiffness tensor components of a tungsten polycrystal calculated according to different grain-interaction models. The same results are obtained for all graininteraction models.

\begin{tabular}{lccccc}
\hline $\mathrm{W}$ & $C_{11}^{S}(\mathrm{GPa})$ & $C_{33}^{S}(\mathrm{GPa})$ & $C_{44}^{S}(\mathrm{GPa})$ & $C_{12}^{S}(\mathrm{GPa})$ & $C_{13}^{S}(\mathrm{GPa})$ \\
\hline All models & 502.32 & 502.32 & 151.51 & 199.29 & 199.29 \\
\hline
\end{tabular}

Table 3. Stiffness tensor components of a copper polycrystal calculated according to different grain-interaction models.

\begin{tabular}{lccccc}
\hline $\mathrm{Cu}$ & $C_{11}^{S}(\mathrm{GPa})$ & $C_{33}^{S}(\mathrm{GPa})$ & $C_{44}^{S}(\mathrm{GPa})$ & $C_{12}^{S}(\mathrm{GPa})$ & $C_{13}^{S}(\mathrm{GPa})$ \\
\hline VW & 202.39 & 198.88 & 47.16 & 102.65 & 106.16 \\
EK (disc) & 202.16 & 199.43 & 47.41 & 103.15 & 105.89 \\
EK (sphere) & 201.30 & 201.30 & 48.17 & 104.95 & 104.95 \\
EK (needle) & 200.84 & 202.15 & 48.49 & 105.84 & 104.52 \\
iVW & 200.80 & 204.08 & 49.56 & 106.84 & 103.56 \\
\hline
\end{tabular}

Values for $A_{i}$ have also been reported in table $1 . A_{i}=1$ is the case of intrinsic elastic isotropy. $A_{i}<1$ indicates that the $\langle h h h\rangle$ direction is softer than the $\langle 00 l\rangle$ direction, whereas $A_{i}>1$ indicates that the $\langle h h h\rangle$ direction is stiffer than the $\langle 00 l\rangle$ direction (this is the case for copper and iron).

\subsection{Mechanical elastic constants}

4.1.1. Tungsten. The components of the mechanical stiffness tensor of a tungsten polycrystal, as calculated according to the VW model, the iVW model and the EK model involving two extreme grain morphologies (flat-disc shaped grains, $\eta=0.1$, and needle-shaped grains, $\eta=10$ ) have been gathered in table 2 .

Tungsten is an intrinsically (practically) elastically isotropic material, since its anisotropy factor $A_{i}$ is equal to 1 . As a consequence, the mechanical elastic properties are insensitive to the grain interaction. Hence, the same isotropic mechanical stiffness tensor is obtained for all types of grain-interaction.

4.1.2. Copper and iron. The components of the mechanical stiffness tensor of copper and iron polycrystals, as calculated according to the VW model, the iVW model and the Eshelby-Kröner (EK) model involving two extreme grain 
Table 4. Stiffness tensor components of an iron polycrystal calculated according to different grain-interaction models.

\begin{tabular}{lccccc}
\hline $\mathrm{Fe}$ & $C_{11}^{S}(\mathrm{GPa})$ & $C_{33}^{S}(\mathrm{GPa})$ & $C_{44}^{S}(\mathrm{GPa})$ & $C_{12}^{S}(\mathrm{GPa})$ & $C_{13}^{S}(\mathrm{GPa})$ \\
\hline VW & 274.30 & 270.54 & 81.24 & 106.06 & 109.83 \\
EK (disc) & 274.06 & 271.12 & 81.49 & 106.60 & 109.54 \\
EK (sphere) & 273.12 & 273.12 & 82.29 & 108.54 & 108.54 \\
EK (needle) & 272.62 & 274.08 & 82.64 & 109.52 & 108.06 \\
iVW & 272.77 & 276.32 & 83.91 & 110.50 & 106.94 \\
\hline
\end{tabular}

morphologies (flat-disc shaped grains, $\eta=0.1$, and needle-shaped grains, $\eta=10$ ) have been gathered in tables 3 and 4 .

Copper and iron exhibit pronounced single-crystal elastic anisotropy and, as a consequence, the components of the mechanical stiffness tensor are also sensitive to grain interaction. For the Eshelby-Kröner model with a spherical grain shape $(\eta=1)$, an obviously isotropic stiffness tensor, with only two independent components $\left(C_{11}^{S}, C_{12}^{S}\right)$, is obtained. It holds that:

$$
C_{44}^{S}=C_{55}^{S}=C_{66}^{S}=\frac{1}{2}\left(C_{11}^{S}-C_{12}^{S}\right)
$$

thus, as expected, $C_{44}^{S}$ is not an independent component in this case.

For the VW and iVW models, transverse elastic isotropy occurs and, in this case, the macroscopic stiffness tensor consists of five independent components $\left(C_{11}^{S}, C_{12}^{S}, C_{13}^{S}, C_{33}^{S}, C_{44}^{S}\right)$; equation (49) remaining true only for $C_{66}^{S}$. The transverse isotropy reflects the rotational symmetry of the grain-interaction assumptions (cf. equations (11) and (12) for the VW model and equations (15) and (16) for the iVW model).

Transverse elastic isotropy also occurs for non-spherical grain shapes $(\eta \neq 1)$. The transverse isotropy reflects the rotational symmetry of the grainshape texture with respect to the surface normal of the specimen. Hence, an isotropic grain interaction occurs for the case of spherical inclusions, but an anisotropic (i.e. direction-dependent) grain interaction occurs if a grain-shape texture is present.

For copper, the calculated components of the mechanical stiffness tensor are shown in figure 2 as a function of the crystallite aspect ratio $\eta$. The values obtained employing the VW and iVW models have also been indicated. As the grain shape approaches the limit of flat-disc shaped grains $(\eta \rightarrow 0)$, the components of the stiffness tensor tend to the corresponding VW values. On the other hand, in the limit of needle-shaped grains $(\eta \rightarrow \infty)$, the components tend towards the corresponding iVW values, but much less obviously, as for the VW values for $\eta \rightarrow 0$. These findings can be summarized as follows:

$$
\begin{aligned}
& \lim _{\eta \rightarrow 0}\left(\mathbf{C}^{S}\right)_{\mathrm{EK}} \approx\left(\mathbf{C}^{S}\right)_{\mathrm{VW}}, \\
& \lim _{\eta \rightarrow \infty}\left(\mathbf{C}^{S}\right)_{\mathrm{EK}} \rightarrow\left(\mathbf{C}^{S}\right)_{\mathrm{iVW}} .
\end{aligned}
$$



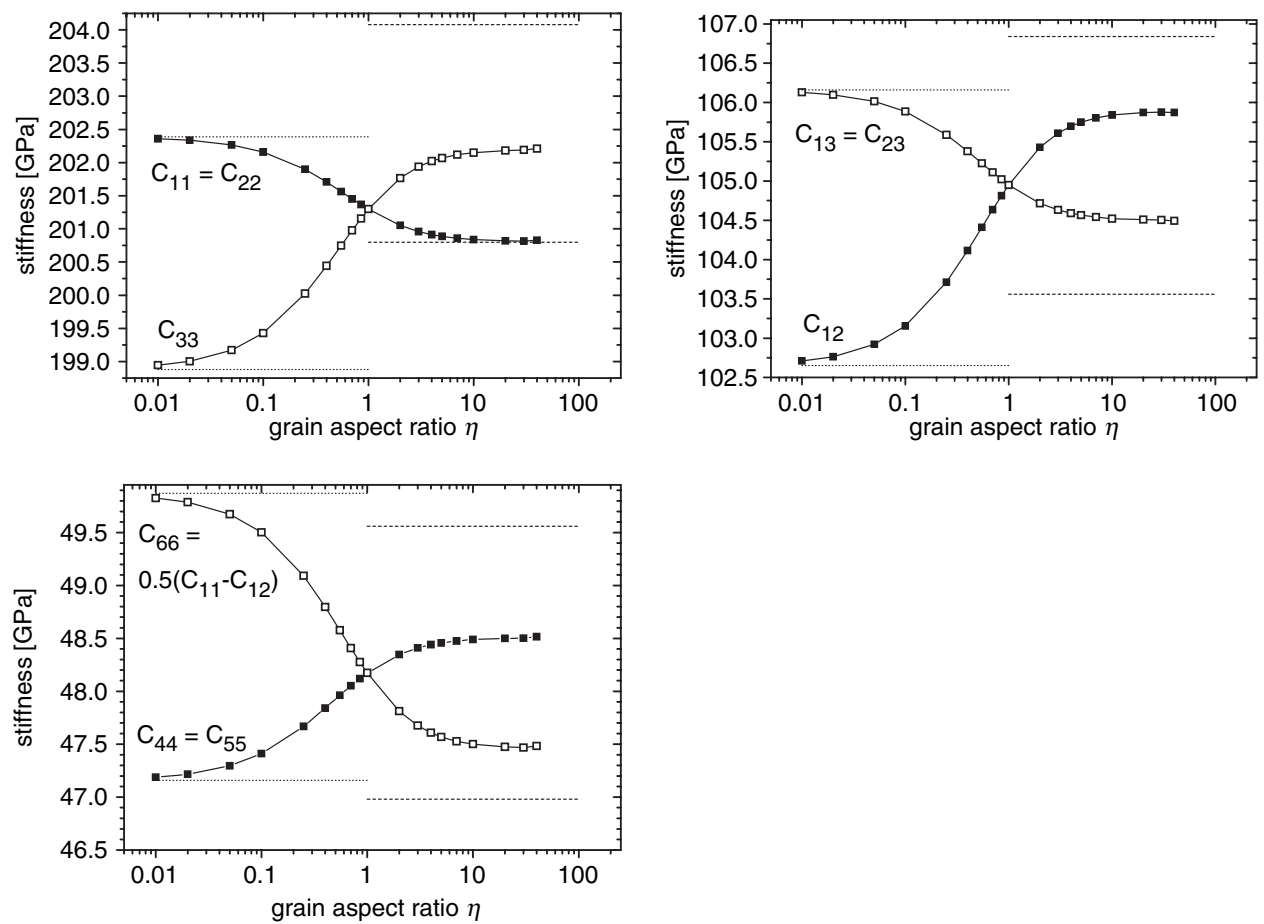

Figure 2. Components of the mechanical stiffness tensor of an untextured copper polycrystal as a function of the grain-aspect ratio, calculated employing the EshelbyKröner model for an ideal grain shape texture. Note that the stiffness tensor presents transverse elastic isotropy (i.e. rotational symmetry with respect to the $z$-axis) for $\eta \neq 1$ and isotropy for $\eta=1$. For comparison, the corresponding values calculated with the Vook-Witt model (dotted line, for $\eta \leq 1$ ) and the inverse Vook-Witt model (dashed line, for $\eta \geq 1$ ) are also shown.

Subscripts have been attached to the stiffness tensor to specify the graininteraction model. The symbol $\approx$ (approximately equal) is used, instead of $=$, to express, that, within the investigated range of grain shapes for the case $\eta \rightarrow 0$, within numerical accuracy, the values are equal to the values obtained using the VW model for the same material. The symbol $\rightarrow$ has been used for the case $\eta \rightarrow \infty$ to express that the values approach the values obtained using the iVW model for the same material, but still differ significantly.

Similar findings have been obtained in an earlier work. A physical interpretation and mathematical explanation of these similarities is given in [5].

\subsection{Diffraction (X-ray) stress factors}

The effect of the grain interaction on the diffraction (X-ray) stress factors will be demonstrated in the following using plots of $\varepsilon(h k l, \varphi, \psi)$ versus $\sin ^{2} \psi$ (so-called 


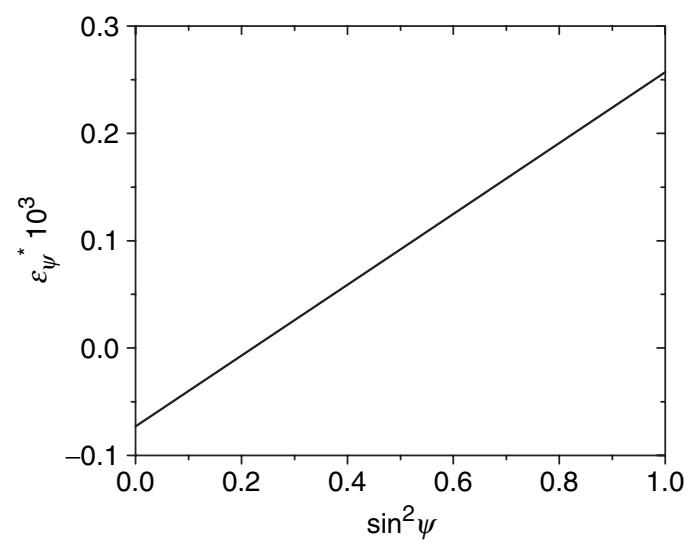

Figure 3. $\operatorname{Sin}^{2} \psi$ plot for a tungsten polycrystal subjected to a uniaxial tensile stress calculated according to different grain-interaction models: Vook-Witt, inverse Vook-Witt and Eshelby-Kröner (with $\eta=0.1$ (discs), $\eta=1$ (spheres), $\eta=10$ (needles)). The same result is obtained for all models and all reflections.

' $\sin ^{2} \psi$-plots'), assuming that the polycrystal considered is subjected to a uniaxial state of stress:

$$
\left\langle\boldsymbol{\sigma}^{S}\right\rangle=\left(\begin{array}{ccc}
\left\langle\sigma_{11}^{S}\right\rangle & 0 & 0 \\
0 & 0 & 0 \\
0 & 0 & 0
\end{array}\right)
$$

with $\left\langle\sigma_{11}^{S}\right\rangle=100 \mathrm{MPa}$. In this case, equation (4) can be simplified to:

$$
\varepsilon(h k l, \varphi, \psi)=\left(F_{11}(\varphi, \psi, h k l)\right)\left\langle\sigma_{11}^{S}\right\rangle .
$$

The lattice strain $\varepsilon(h k l, \varphi, \psi)$ will be plotted for $\varphi=0$ in the following.

4.2.1. Tungsten. A $\sin ^{2} \psi$-plot for a tungsten polycrystal subjected to a uniaxial state of stress $(100 \mathrm{MPa})$ is shown in figure 3.

The same straight line obtained in the $\sin ^{2} \psi$-plot is independent of the type of grain interaction or $h k l$ reflection chosen. This is a consequence of the intrinsic elastic isotropy, which makes the diffraction stress factors (as well as the mechanical elastic constants; cf. section 4.1) insensitive to grain interaction.

4.2.2. Copper and iron. In figures 4 and $5, \sin ^{2} \psi$-plots calculated according to the VW, iVW and EK (for the case of spherical grains, $\eta=1$, and for grain-shape textures with $\eta=0.1$ and $\eta=10$, cf. section 2) models for crystallographically untextured copper and iron polycrystals subjected to uniaxial states of stress (100 MPa) are shown.

For spherical grain shape, linear $\sin ^{2} \psi$-plots are obtained for all reflections, indicating the occurrence of macroscopic/mechanical isotropy. However, for the VW model, iVW model and a grain-shape texture, distinct 

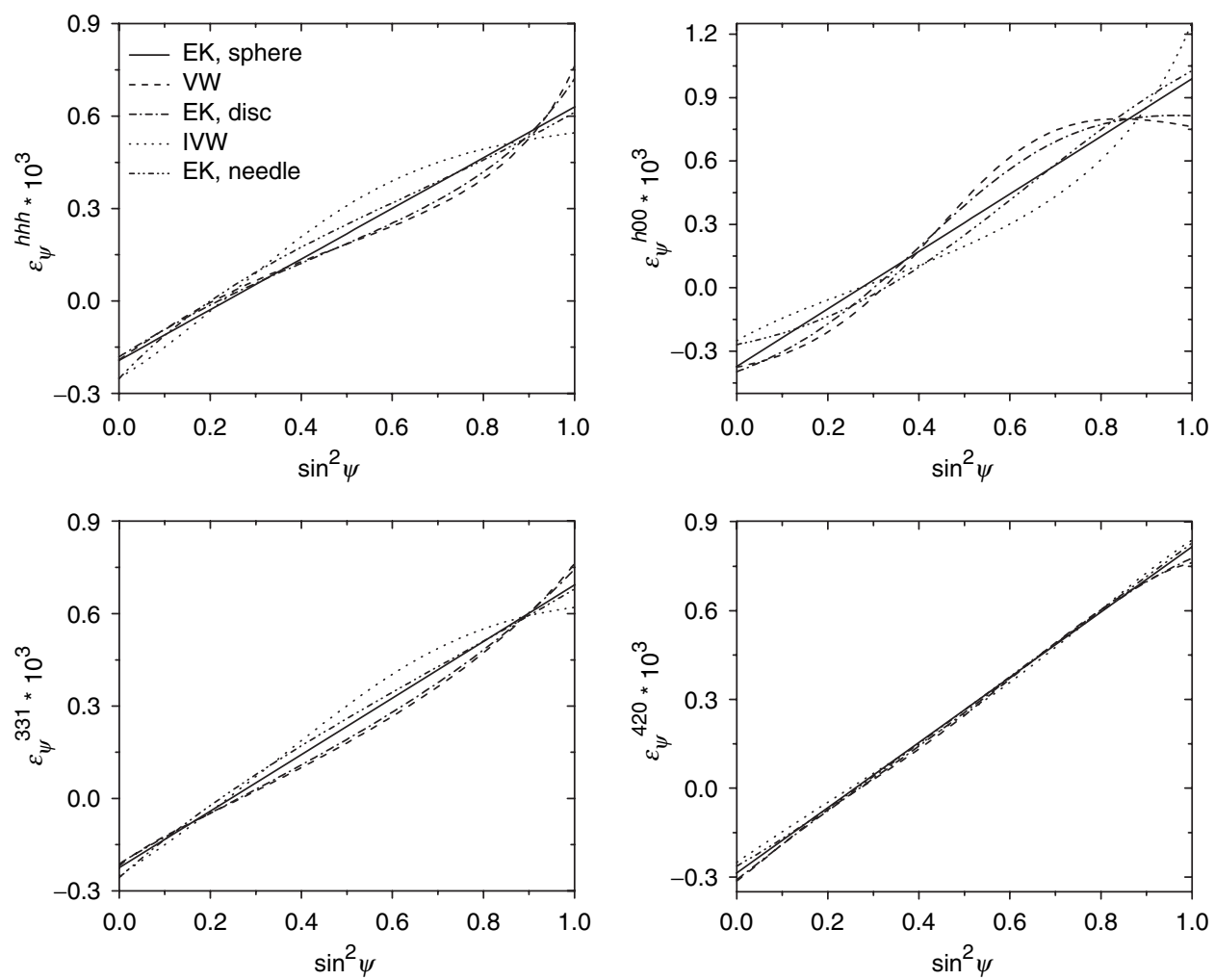

Figure 4. $\operatorname{Sin}^{2} \psi$ plots for a copper polycrystal subjected to a uniaxial tensile stress calculated according to different grain-interaction models: Vook-Witt, inverse Vook-Witt and Eshelby-Kröner model (with $\eta=0.1$ (discs), $\eta=1$ (spheres), $\eta=10$ (needles)).

curvature occurs in the $\sin ^{2} \psi$-plots, most pronouncedly for the 200 reflections. Figures 4 and 5 also demonstrate that the effect of the grain-interaction on the diffraction elastic constants can be stronger than the effect on the mechanical elastic constants (cf. section 4.1).

The occurrence of non-linear $\sin ^{2} \psi$-plots corresponds to the occurrence of macroscopic elastic anisotropy. This is well known in the context of the diffraction stress analysis of crystallographically textured specimens, where the occurrence of crystallographic texture leads to mechanical anisotropy and non-linear $\sin ^{2} \psi$-plots. It was shown very recently that non-linear $\sin ^{2} \psi$-plots could occur in general for macroscopically, elastically anisotropic specimens, independent of the occurrence of crystallographic texture [4].

Striking similarities among the $\sin ^{2} \psi$-plots, calculated with the different graininteraction models, can be observed. The VW model results in $\operatorname{similar}^{2} \sin ^{2} \psi$-plots as the EK model for flat-disc shaped grains $(\eta=0.1)$, whereas the $\sin ^{2} \psi$-plots obtained from the iVW model exhibit similarities (but much less obviously) with the corresponding plots obtained from the EK model for needle-shaped inclusions $(\eta=10)$. These findings correspond to the results obtained for the mechanical elastic 

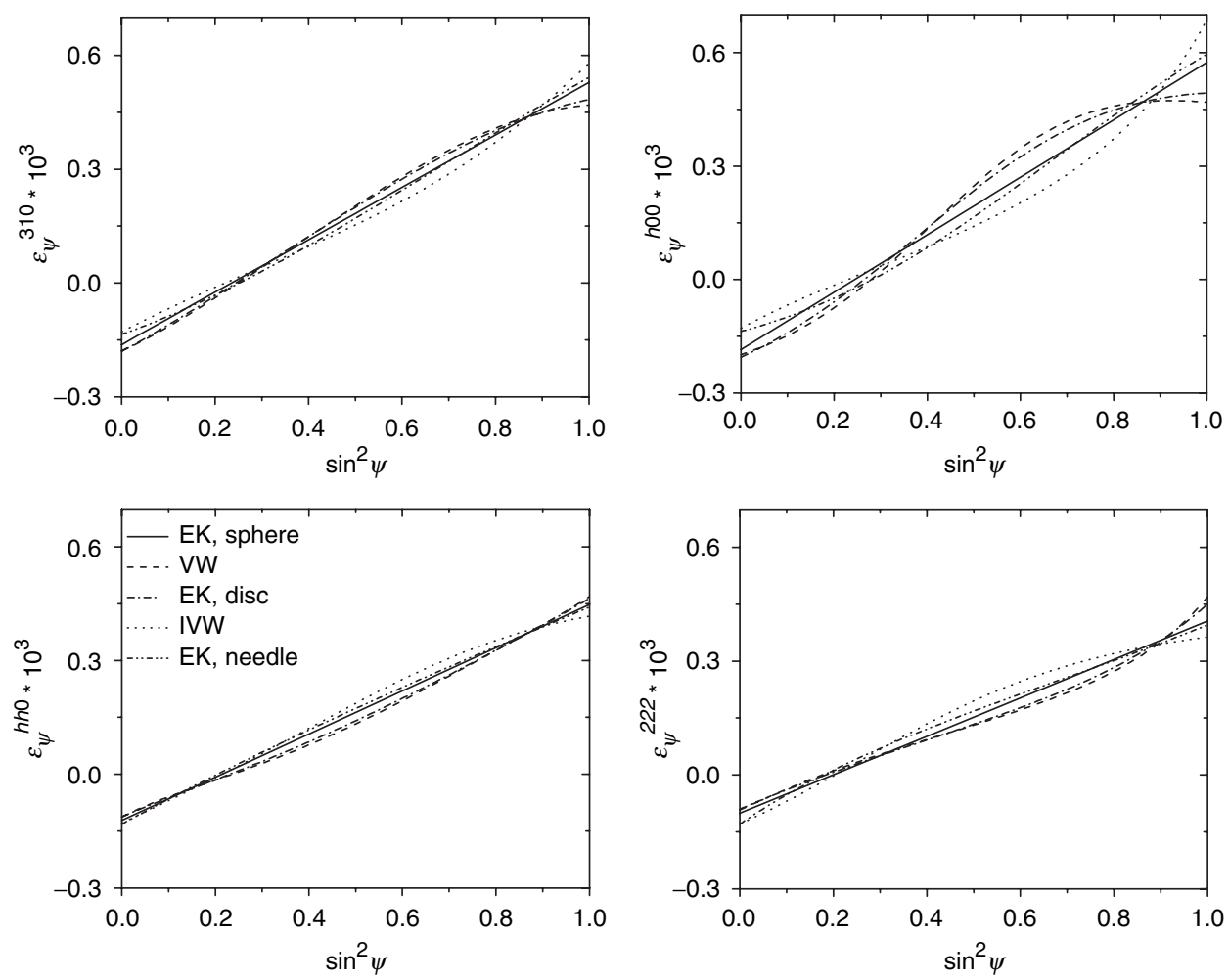

Figure 5. $\operatorname{Sin}^{2} \psi$ plots for a iron polycrystal subjected to a uniaxial tensile stress calculated according to different grain-interaction models: Vook-Witt, inverse Vook-Witt and Eshelby-Kröner (with $\eta=0.1$ (discs), $\eta=1$ (spheres), $\eta=10$ (needles)).

constants in section 4.1. Similar findings have been obtained in an earlier work. Also, a physical interpretation and mathematical explanation of these similarities have been given recently. The physical interpretation also makes the much less pronounced agreement of the iVW and EK model for needle-shaped inclusions, compared to the agreement between the VW model and the EK model for flat-disc shaped grains, plausible [5].

\section{Summary}

- Generalizations of the elastic grain-interaction assumptions of the Vook-Witt and inverse Vook-Witt models allow the calculation of the full mechanical stiffness (and compliance) tensors and of all diffraction stress factors, whereas previously only two mechanical elastic constants and only the sum of two diffraction (X-ray) stress factors could have been calculated.

- The direction-dependent grain-interaction assumptions, employed in the Vook-Witt and inverse Vook-Witt models, are reflected by the occurrence 
of macroscopic elastic anisotropy (transverse isotropy) for the mechanical compliance tensor, i.e. five independent components occur. The effect of the elastic grain interaction on the mechanical elastic constants is generally weak.

- The direction-dependent grain-interaction assumptions employed in the Vook-Witt and inverse Vook-Witt models are also reflected by the occurrence of non-linear $\sin ^{2} \psi$-plots. The effect of elastic grain interaction on the diffraction stress factors can be large: distinct curvature can occur in $\sin ^{2} \psi$-plots, as has been demonstrated for the case of a uniaxial stress state. Diffraction stress analysis is, therefore, a sensitive tool to investigate elastic grain interaction.

- The mechanical elastic constants and diffraction stress factors (represented in the form of $\sin ^{2} \psi$-plots) have been compared with corresponding values calculated employing the Eshelby-Kröner model, considering ideal morphological (grain-shape) textures. Similarities between the Vook-Witt and the Eshelby-Kröner model occur where a polycrystal, consisting of flat discshaped grains with their principal shape axis aligned with the specimen surface normal, is considered for the Eshelby-Kröner model. These similarities concern the diffraction and mechanical elastic constants. Such similarities among the inverse Vook-Witt and the Eshelby-Kröner model also occur (but much less obviously, on a more qualitative level) where a polycrystal, consisting of needle-shaped grains with their principal shape (needle) axis aligned with the specimen surface normal, is considered for the EshelbyKröner model. These findings are in line with previous results [5].

- Hence, even though the Vook-Witt and inverse Vook-Witt models have been developed to express, in particular, the effect of elastic surface anisotropy of bulk polycrystals and thin films, they are capable of modelling the effect of special, limiting morphological (grain-shape) textures on the elastic grain interaction. Thus, time-consuming numerical calculations, as required in the application of the Eshelby-Kröner model, can be replaced by less elaborate calculations, according to the Vook-Witt (for flat-disc shaped grain morphology) or inverse Vook-Witt (for needle-shaped grain morphology) models (for the latter, with a qualitative agreement only; see above). This can greatly simplify numerical algorithms for the calculation of elastic and, possibly, plastic grain interaction.

\section{Acknowledgments}

The authors express their gratitude to Professor Dr Ir. E. J. Mittemeijer for constructive discussions.

\section{References}

[1] P.D. Chinh, Phil. Mag. 86205 (2006).

[2] E. Kröner, J. Phys. F: Metal Phys. 82261 (1978). 
[3] V. Hauk (Editor), Structural and Residual Stress Analysis by Nondestructive Methods (Elsevier, Amsterdam, 1997).

[4] U. Welzel and E.J. Mittemeijer, J. Appl. Phys. 939001 (2003).

[5] U. Welzel, S. Fréour and E.J. Mittemeijer, Phil. Mag. 852391 (2005).

[6] U. Welzel, J. Ligot, P. Lamparter, et al., J. Appl. Cryst. 381 (2005).

[7] W. Voigt, Lehrbuch der Kristallphysik (Teubner, Leipzig, 1910).

[8] A. Reuss, Z. Angew. Math. Mech. 949 (1929).

[9] H. Neerfeld, Mitt. K.-Wilh.-Inst. Eisenforschg. 2461 (1942).

[10] R. Hill, Proc. Phys. Soc. London A 65349 (1952).

[11] E. Kröner, Z. Phys. 151504 (1958).

[12] M. van Leeuwen, J.-D. Kamminga and E.J. Mittemeijer, J. Appl. Phys. 861904 (1999).

[13] M. Leoni, U. Welzel, P. Lamparter, et al., Phil. Mag. A 81597 (2001).

[14] U. Welzel, M. Leoni and E.J. Mittemeijer, Phil. Mag. 83603 (2003).

[15] U. Welzel, M. Leoni and E.J. Mittemeijer, in Diffraction Analysis of the Microstructure of Materials, edited by E.J. Mittemeijer and P. Scardi (Springer, Berlin, 2004), pp. 363-390.

[16] R.W. Vook and F. Witt, J. Appl. Phys. 362169 (1965).

[17] N. Koch, U. Welzel, H. Wern and E.J. Mittemeijer, Phil. Mag. 843547 (2004).

[18] C. Giacovazzo, H.L. Monaco, D. Vioterbo, et al., Fundamentals of Crystallography (Oxford Science, Oxford, 1998).

[19] J.M. Sprauel and L.Castex, Mater. Sci. Forum 79/82 143 (1991).

[20] R.-J. Roe and W.R. Krigbaum, J. Chem. Phys. 402608 (1964).

[21] M.A. Meyers and K.K. Chawla, Mechanical Metallurgy, Principles and Applications (Prentice-Hall, Englewood Cliffs, NJ, 1984), p. 57.

\section{Appendix A: Calculation of stress and strain tensor components not defined directly by the grain-interaction assumptions}

\section{A.1. Vook-Witt model}

From the stress and strain tensor components equal to the mechanical averages (cf. equations (11) and (12)), the unknown components can be calculated as follows:

After defining the vector $\mathbf{x}$ of which the components are the unknown components of the stress tensor $\boldsymbol{\sigma}^{S}$ :

$$
\mathbf{x}=\left(\begin{array}{c}
\sigma_{11}^{S} \\
\sigma_{12}^{S} \\
\sigma_{22}^{S}
\end{array}\right)
$$

the unknown stress tensor components $\left(\sigma_{11}^{S}, \sigma_{12}^{S}, \sigma_{22}^{S}\right)$ can be obtained from:

$$
\mathbf{x}=\mathbf{A}^{-1} \mathbf{b}
$$

with:

$$
\begin{aligned}
& A_{11}=1-c_{1133}^{S} S_{3311}^{S}-2 c_{1113}^{S} s_{1311}^{S}-2 c_{1123}^{S} s_{2311}^{S} \\
& A_{12}=-2 c_{1133}^{S} s_{3312}^{S}-4 c_{1113}^{S} s_{1312}^{S}-4 c_{1123}^{S} s_{2312}^{S}
\end{aligned}
$$




$$
\begin{aligned}
& A_{13}=-c_{1133}^{S} S_{3322}^{S}-2 c_{1113}^{S} S_{1322}^{S}-2 c_{1123}^{S} s_{2322}^{S} \\
& A_{21}=-c_{1233}^{S} S_{3311}^{S}-2 c_{1213}^{S} s_{1311}^{S}-2 c_{1223}^{S} s_{2311}^{S} \\
& A_{22}=1-2 c_{1233}^{S} S_{3312}^{S}-4 c_{1213}^{S} s_{1312}^{S}-4 c_{1223}^{S} S_{2312}^{S} \\
& A_{23}=-c_{1233}^{S} s_{3322}^{S}-2 c_{1213}^{S} s_{1322}^{S}-2 c_{1223}^{S} s_{2322}^{S} \\
& A_{31}=-c_{2233}^{S} S_{3311}^{S}-2 c_{2213}^{S} s_{1311}^{S}-2 c_{2223}^{S} s_{2311}^{S} \\
& A_{32}=-2 c_{2233}^{S} S_{3312}^{S}-4 c_{2213}^{S} s_{1312}^{S}-4 c_{2223}^{S} S_{2312}^{S} \\
& A_{33}=1-c_{2233}^{S} S_{3322}^{S}-2 c_{2213}^{S} S_{1322}^{S}-2 c_{2223}^{S} S_{2322}^{S}
\end{aligned}
$$

and

$$
\mathbf{b}=\left(\begin{array}{l}
c_{1111}^{S}\left\langle\varepsilon_{11}^{S}\right\rangle+c_{1122}^{S}\left\langle\varepsilon_{22}^{S}\right\rangle+2 c_{112}^{S}\left\langle\varepsilon_{12}^{S}\right\rangle+c_{1133}^{S} \xi_{1}+2 c_{1113}^{S} \xi_{2}+2 c_{1312}^{S} \xi_{3} \\
c_{1211}^{S}\left\langle\varepsilon_{11}^{S}\right\rangle+c_{1222}^{S}\left\langle\varepsilon_{22}^{S}\right\rangle+2 c_{1212}^{S}\left\langle\varepsilon_{12}^{S}\right\rangle+c_{1233}^{S} \xi_{1}+2 c_{1213}^{S} \xi_{2}+2 c_{2312}^{S} \xi_{3} \\
c_{2211}^{S}\left\langle\varepsilon_{11}^{S}\right\rangle+c_{2222}^{S}\left\langle\varepsilon_{22}^{S}\right\rangle+2 c_{2212}^{S}\left\langle\varepsilon_{12}^{S}\right\rangle+c_{2233}^{S} \xi_{1}+2 c_{2213}^{S} \xi_{2}+2 c_{3312}^{S} \xi_{3}
\end{array}\right)
$$

with:

$$
\begin{aligned}
& \xi_{1}=s_{3333}^{S}\left\langle\sigma_{33}^{S}\right\rangle+2 s_{3313}^{S}\left\langle\sigma_{13}^{S}\right\rangle+s_{3323}^{S}\left\langle\sigma_{23}^{S}\right\rangle, \\
& \xi_{2}=s_{1333}^{S}\left\langle\sigma_{33}^{S}\right\rangle+2 s_{1313}^{S}\left\langle\sigma_{13}^{S}\right\rangle+s_{1323}^{S}\left\langle\sigma_{23}^{S}\right\rangle, \\
& \xi_{3}=s_{2333}^{S}\left\langle\sigma_{33}^{S}\right\rangle+2 s_{2313}^{S}\left\langle\sigma_{13}^{S}\right\rangle+s_{2323}^{S}\left\langle\sigma_{23}^{S}\right\rangle .
\end{aligned}
$$

Then, the unknown strain tensor components can be obtained employing Hooke's law (equation (10)).

\section{A.2. Inverse Vook-Witt model}

From the stress and strain tensor components equal to the mechanical averages (cf. equations (15) and (16)), the unknown components can be calculated as follows:

After defining the vector $\mathbf{x}$ of which the components are the unknown components of the stress tensor $\boldsymbol{\sigma}^{S}$ :

$$
\mathbf{x}=\left(\begin{array}{c}
\sigma_{13}^{S} \\
\sigma_{23}^{S} \\
\sigma_{33}^{S}
\end{array}\right)
$$

the unknown stress tensor components $\left(\sigma_{13}^{S}, \sigma_{23}^{S}, \sigma_{33}^{S}\right)$ can be obtained from:

$$
\mathbf{x}=\mathbf{A}^{-1} \mathbf{b}
$$

with:

$$
\begin{gathered}
A_{11}=1-2 c_{1311}^{S} s_{1113}^{S}-2 c_{1322}^{S} s_{2213}^{S}-4 c_{1312}^{S} s_{1213}^{S} \\
A_{12}=-2 c_{1311}^{S} s_{1123}^{S}-2 c_{1322}^{S} s_{2223}^{S}-4 c_{1312}^{S} s_{1223}^{S}
\end{gathered}
$$




$$
\begin{gathered}
A_{13}=-c_{1311}^{S} s_{1133}^{S}-c_{1322}^{S} S_{2233}^{S}-2 c_{1312}^{S} s_{1233}^{S} \\
A_{21}=-2 c_{2311}^{S} s_{1113}^{S}-2 c_{2322}^{S} s_{2213}^{S}-4 c_{2312}^{S} s_{1213}^{S} \\
A_{22}=1-2 c_{2311}^{S} s_{1123}^{S}-2 c_{2322}^{S} S_{2223}^{S}-4 c_{2312}^{S} S_{1223}^{S} \\
A_{23}=-c_{2311}^{S} s_{1133}^{S}-c_{2322}^{S} S_{2233}^{S}-2 c_{2312}^{S} s_{1233}^{S} \\
A_{31}=-2 c_{3311}^{S} s_{1113}^{S}-2 c_{3322}^{S} s_{2213}^{S}-4 c_{3312}^{S} s_{1213}^{S} \\
A_{32}=-2 c_{3311}^{S} s_{1123}^{S}-2 c_{3322}^{S} s_{2223}^{S}-4 c_{3312}^{S} s_{1233}^{S} \\
A_{33}=1-c_{3311}^{S} s_{1133}^{S}-c_{3322}^{S} s_{2223}^{S}-2 c_{3312}^{S} s_{1233}^{S}
\end{gathered}
$$

and

$$
\mathbf{b}=\left(\begin{array}{l}
c_{1333}^{S}\left\langle\varepsilon_{33}^{S}\right\rangle+2 c_{1313}^{S}\left\langle\varepsilon_{13}^{S}\right\rangle+2 c_{1323}^{S}\left\langle\varepsilon_{23}^{S}\right\rangle+c_{1311}^{S} \xi_{1}+c_{1322}^{S} \xi_{2}+2 c_{1312}^{S} \xi_{3} \\
c_{2333}^{S}\left\langle\varepsilon_{33}^{S}\right\rangle+2 c_{2313}^{S}\left\langle\varepsilon_{13}^{S}\right\rangle+2 c_{2323}^{S}\left\langle\varepsilon_{23}^{S}\right\rangle+c_{3211}^{S} \xi_{1}+c_{2322}^{S} \xi_{2}+2 c_{2312}^{S} \xi_{3} \\
c_{3333}^{S}\left\langle\varepsilon_{33}^{S}\right\rangle+2 c_{3313}^{S}\left\langle\varepsilon_{13}^{S}\right\rangle+2 c_{3323}^{S}\left\langle\varepsilon_{23}^{S}\right\rangle+c_{3311}^{S} \xi_{1}+c_{3322}^{S} \xi_{2}+2 c_{3312}^{S} \xi_{3}
\end{array}\right)
$$

with:

$$
\begin{aligned}
& \xi_{1}=s_{1111}^{S}\left\langle\sigma_{11}^{S}\right\rangle+s_{1122}^{S}\left\langle\sigma_{22}^{S}\right\rangle+2 s_{1112}^{S}\left\langle\sigma_{12}^{S}\right\rangle, \\
& \xi_{2}=s_{2211}^{S}\left\langle\sigma_{11}^{S}\right\rangle+s_{2222}^{S}\left\langle\sigma_{22}^{S}\right\rangle+2 s_{2212}^{S}\left\langle\sigma_{12}^{S}\right\rangle, \\
& \xi_{3}=s_{1211}^{S}\left\langle\sigma_{11}^{S}\right\rangle+s_{1222}^{S}\left\langle\sigma_{22}^{S}\right\rangle+2 s_{1212}^{S}\left\langle\sigma_{12}^{S}\right\rangle .
\end{aligned}
$$

Then, the unknown strain tensor components can be obtained employing Hooke's law (equation (10)).

\section{Appendix B: Loading states for the case of orthorhombic texture symmetry}

For crystallographic texture, more than five independent components of the mechanical stiffness (and compliance) tensor can occur. For orthorhombic texture symmetry, for example, nine independent components of the mechanical stiffness (and compliance) tensor occur and, then, the following four loading states are sufficient for a calculation of these nine tensor components:

$$
\left\langle\varepsilon^{S}\right\rangle=\left(\begin{array}{ccc}
\left\langle\varepsilon_{11}^{S}\right\rangle & 0 & 0 \\
0 & 0 & 0 \\
0 & 0 & 0
\end{array}\right),
$$




$$
\begin{gathered}
\left\langle\varepsilon^{S}\right\rangle=\left(\begin{array}{ccc}
0 & 0 & 0 \\
0 & \left\langle\varepsilon_{22}^{S}\right\rangle & 0 \\
0 & 0 & 0
\end{array}\right), \\
\left\langle\varepsilon^{S}\right\rangle=\left(\begin{array}{ccc}
0 & 0 & 0 \\
0 & 0 & 0 \\
0 & 0 & \left\langle\varepsilon_{33}^{S}\right\rangle
\end{array}\right), \\
\left\langle\varepsilon^{S}\right\rangle=\left(\begin{array}{ccc}
0 & \left\langle\varepsilon_{12}^{S}\right\rangle & \left\langle\varepsilon_{13}^{S}\right\rangle \\
\left\langle\varepsilon_{12}^{S}\right\rangle & 0 & \left\langle\varepsilon_{23}^{S}\right\rangle \\
\left\langle\varepsilon_{13}^{S}\right\rangle & \left\langle\varepsilon_{23}^{S}\right\rangle & 0
\end{array}\right) .
\end{gathered}
$$

Note that other sets of hypothetical loading states can be conceived, which also allow the evaluation of the full stiffness tensor. 